\title{
Utopie paesaggistiche : dal paesaggio-angelo al paesaggio-spaventapasseri
}

\section{Massimo Quaini}

\section{(2) OpenEdition \\ 1 Journals}

Edizione digitale

URL: http://journals.openedition.org/edl/513

DOI: $10.4000 /$ edl. 513

ISSN: 2296-5084

\section{Editore}

Université de Lausanne

\section{Edizione cartacea}

Data di pubblicazione: 15 mai 2013

Paginazione: 293-304

ISBN: 978-2-940331-32-1

ISSN: 0014-2026

Notizia bibliografica digitale

Massimo Quaini, «Utopie paesaggistiche : dal paesaggio-angelo al paesaggio-spaventapasseri », Études de lettres [Online], 1-2 | 2013, online dal 15 mai 2016, consultato il 21 décembre 2020. URL http://journals.openedition.org/edl/513 ; DOI : https://doi.org/10.4000/edl.513 


\section{UTOPIE PAESAGGISTICHE: DAL PAESAGGIO-ANGELO AL PAESAGGIO-SPAVENTAPASSERI}

L'auteur réfléchit sur quelques allégories et métaphores utilisées par des artistes, des philosophes et des écrivains pour interpréter le paysage. Ces images figurées constituent des outils de compréhension souvent plus affutés que ceux qui ont été élaborés par les urbanistes, les historiens et les géographes. Ce constat suggère la nécessité pour les géographes et les urbanistes de retrouver et de consolider leur lien avec l'art et la littérature. Pour la Ligurie, en particulier, l'apport intellectuel d'écrivains comme Calvino et Biamonti est fondamentale.

Che cosa può unire fra loro oggetti difformi come l'ultimo libro sul paesaggio di Salvatore Settis ${ }^{1}$, una campagna fotografica sugli spaventapasseri che si possono ancora incontrare nei paesaggi rurali, l'Angelus Novus di Benjamin e una poesia di Tonino Guerra? Troveremo forse, alla fine, una risposta anche grazie a un'enigmatica «mappa» di Francesco Casorati intitolata La morte del generale $(1973)^{2}$, che si potrebbe assumere come sintesi grafica del percorso che ora vi propongo.

Non date troppo importanza a questa mia breve relazione introduttiva. Anche per lasciare spazio a quanti affronteranno le diverse sfaccettature del paesaggio, mi limito a ricavare qualche suggestione da alcuni simboli, allegorie e pregnanti metafore del nostro tempo, veicolati

I. S. Settis, Paesaggio, costituzione, cemento. URL: http://www.einaudi.it/layout/ set/popup/content/view/popup/360/(tipo)/10/(isbn)/978880619871, consultato il 15.12.2012.

2. URL: http://www.arte2000.net/artisti/casorati/opere/1970/1970.htm, consultato il 15.12.2012. 
soprattutto dal linguaggio figurato. Mi avvio su questa strada anche per fare un piccolo omaggio a Francesco Biamonti, sempre attento (come Italo Calvino) al rapporto fra arte e letteratura.

L'immagine stampata sulla copertina del libro di Salvatore Settis nella sua didascalia recita: "Paesaggio antropomorfo, olio su tela, prima metà del XVIII secolo.» Quella che a una prima occhiata sembra essere una delle tante vedute campestri del Settecento diventa un esercizio raffinato di antropomorfismo paesaggistico che si rivela solo se si fa ruotare il libro e l'immagine: allora il paesaggio boscoso si rivela essere il volto umano di una sorta di genio della contrada. Come dire meglio che il paesaggio, anche quando appare essenzialmente come vegetazione e rilievo montuoso, è natura umanizzata, trasformata dal lavoro dell'uomo?

Il senso è così evidente che Settis non ha bisogno di dire quale sia il significato del quadro. Sembra piuttosto invitare il lettore a scoprirlo offrendogli molti materiali interpretativi. Se infatti volessimo darne un'interpretazione ancora più pertinente e geograficamente estensiva si potrebbe dire che il paesaggio è "il volto amato della patria» ovvero, come diceva Benedetto Croce, citato da Settis, "non essere altro che la rappresentazione materiale e visibile della patria, coi suoi caratteri fisici particolari [...] con gli aspetti molteplici e vari del suo suolo, quali si sono formati e son pervenuti a noi attraverso la lenta successione dei secoli ». La citazione è del $1920^{3}$ e fa soprattutto riferimento alla filosofia tedesca e a una visione del paesaggio che potrebbe già risalire a Goethe.

Il paesaggio, dunque, come allegoria dell'uomo, del suo identificarsi nell'ambiente al punto che i boschi, i campi, le sedi umane e il suolo diventano la "pelle» e il cuore, la fisionomia e la personalità-identità di un popolo, di una società che vive in simbiosi con il territorio. Di fatto è questa l'immagine prescrittiva che Settis intende dare al suo lettore per contrastare un presente fatto di alluvioni cementizie e di consumo abnorme di suolo che stanno cancellando il paesaggio italiano.

Il paesaggio è dunque innanzitutto allegoria. Per altri è metafora. Su questo terreno ci si potrebbe sbizzarrire a lungo, a patto di non dimenticare che il paesaggio non è solo una figura retorica, ma

3. Disegno di Legge n. 204, Presentato dal Ministro dell'Istruzione Pubblica (CROCE) nella tornata del 25 settembre 1920, Per la tutela delle bellezze naturali e degli immobili di particolare interesse storico, URL: http://rivista.ssef.it/site.php?page=200409 13091214766\&edition=2010-02-01, consultato il 15.12.2012. 
è anche qualcosa che esiste nella realtà al di là delle nostre parole e rappresentazioni.

Ma rimaniamo ancora per un momento sulla allegoria. La definizione più semplice dice: «Narrazione o Rappresentazione che vuole dire qualcosa di diverso da quello che significa o rappresenta alla lettera.» L'allegoria ci può dunque aiutare ad andare al di là delle parole e del loro significato letterale. Non per caso la ricerca allegorica era il metodo preferito di Walter Benjamin. La seconda immagine che vi propongo è allora l'Angelus Novus 4 (1920) di Paul Klee, che per Benjamin e i suoi editori aveva una carica simbolica così forte da derivarne non solo il nome della rivista progettata nel 1930 che non andò in porto, ma anche il titolo della principale raccolta italiana di scritti riuniti da Renato Solmi nei Saggi Einaudi, nel $1962^{5}$. Solmi vi ricorda giustamente la frase di Benjamin: "le allegorie sono nel regno delle idee ciò che le rovine sono nel regno delle cose: simboli cifrati del passato, che dicono sempre di più, che dicono altro da quello che s'intendeva esprimere con esse.» In altri termini, "l'allegoria è la forma che assume il passato incompreso", in particolare quello rappresentato nell'arte simbolica che, nella riserva inesauribile dei suoi simboli, "non è la più semplice natura ma la storia come natura o la natura come storia» ${ }^{6}$.

Il metodo allegorico come ce lo propone Benjamin è non solo il metodo per decifrare "il passato incompreso" ma anche il metodo che consente di vedere «la storia come natura o la natura come storia»: è il metodo che si fa forte del materialismo storico e della dialettica di Marx che nell'Ideologia tedesca combatte in anticipo le derive idealistiche ed estetizzanti che accompagnano il paesaggio nella sua storia novecentesca.

L'angelogia o scienza degli angeli - a cui appartiene di diritto la serie degli angeli di Klee e per certi versi anche la filosofia di Benjamin (il suo materialismo storico impastato di temi messianici) - è una scienza che si presta a molti equivoci : se ci lasciamo incantare è perché esiste un evidente rapporto fra gli angeli e il paesaggio. Ce lo confermano

4. Conservato al Museo israeliano di Gerusalemme. URL: http://www.english. imjnet.org.il/Media/Uploads/Klee-Paul-Angelus-Novus-192.jpg, consultato il 15.12.2012. Anche in M. Cacciari, L’Angelo necessario, p. 30 sq.

5. W. Benjamin, Angelus novus.

6. Ibid., p. XIX sq. 
Massimo Cacciari ${ }^{7}$ e Giorgio Agamben ${ }^{8}$. Il primo proponendoci, come avvio interpretativo, una bella poesia di Wallace Stevens intitolata L'angelo necessario, il secondo un percorso che ci porta direttamente al linguaggio degli uccelli e degli spaventapasseri, passando attraverso l'opera di Tonino Guerra. Leggiamo insieme L'angelo necessario ${ }^{9}$ :

Io sono l'Angelo della realtà, intravisto un istante sulla soglia.

Non mi seguono stelle in corteo, in me racchiudo l'essere e il conoscere.

Sono uno come voi, e ciò che sono e so, per me come per voi, è la stessa cosa.

Eppure io sono l'Angelo necessario della terra, poiché chi vede me vede di nuovo la terra, libera dai ceppi della mente, dura, caparbia, e chi ascolta me ne ascolta il canto monotono levarsi in liquide lentezze e affiorare in sillabe d'acqua; come un significato che si cerchi per ripetizioni, approssimando [...].

Mi pare evidente che qui si parla di paesaggio e nella sua più giusta definizione filosofica: «in me racchiudo l'essere e il conoscere» (ovvero non possiamo separare il paesaggio-rappresentazione dal paesaggio-realtà). Si parla di paesaggio evocando poeticamente «le liquide lentezze, le sillabe d'acqua", ma si dà anche una bellissima definizione del paesaggio come l'Angelo necessario della Terra, l'entità che libera la terra dai ceppi della mente, dal calcolo razionale e dalla misura del mercato.

In breve, la poesia ci aiuta a capire che l'angelo-paesaggio è necessario in quanto custodisce l'unità perduta dell'essere e del conoscere, l'unità di cui ci parlava anche l'allegoria del paesaggio antropomorfo, fra il paesaggio e ciò che noi siamo e sappiamo ("la stessa cosa»).

L'angelo, dunque, come conoscenza necessaria: "poiché chi vede me vede di nuovo la terra, libera dai ceppi della mente.» Della mente e della storia o meglio di una certa storia: quella a cui si volgono gli occhi spalancati dell'Angelus Novus, secondo l'interpretazione che ne da Walter Benjamin nelle Tesi di filosofia della storia:

7. M. Cacciari, L'angelo necessario.

8. G. Agamben, L'uomo senza contenuto.

9. M. Cacciari, L'angelo necessario, p. 9. 
C'è un quadro di Klee che s'intitola Angelus Novus. Vi si trova un angelo che sembra in atto di allontanarsi da qualcosa su cui fissa lo sguardo. Ha gli occhi spalancati, la bocca aperta, le ali distese. L'angelo della storia deve avere questo aspetto. Ha il viso rivolto al passato. Dove ci appare una catena di eventi, egli vede una sola catastrofe, che accumula senza tregua rovine su rovine e le rovescia ai suoi piedi. Egli vorrebbe ben trattenersi, destare i morti e ricomporre l'infranto. Ma una tempesta spira dal paradiso, che si è impigliata nelle sue ali, ed è così forte che egli non può chiuderle. Questa tempesta lo spinge irresistibilmente nel futuro, a cui volta le spalle, mentre il cumulo delle rovine sale davanti a lui al cielo. Ciò che chiamiamo progresso, è questa tempesta ${ }^{10}$.

Non ci vuole molto acume per interpretare il paesaggio odierno come l'insieme di queste rovine che la catastrofe della storia accumula ai piedi dell'angelo spinto dal vento del progresso verso un futuro di cui si legge un unico scenario: «il cumulo delle rovine che sale al cielo.»

L'angelo di Benjamin, a differenza di quello di Stevens, è un testimone muto che sembra avere ben poco da offrire a noi vittime della tempesta del progresso nella quale si riassume la tragedia della modernità. $\mathrm{Ma}$ un messaggio, un invito pressante c'è e per noi è importante evidenziarlo e praticarlo proprio in questa terra di Biamonti: fare ciò che l'angelo vorrebbe ma non può fare: trattenersi, fermare la corsa folle alla distruzione totale del paesaggio e quindi di noi stessi, "destare i morti " che tanto hanno ancora da dirci e "ricomporre l'infranto».

Giorgio Agamben completa la sua interpretazione con l'immagine di un secondo angelo, la Malinconia di Dürer, che rappresenta l'angelo dell'arte:

Essa rappresenta una creatura alata, seduta in atto di meditare con lo sguardo assorto davanti a sé. Accanto ad essa, giacciono abbandonati al suolo gli utensili della vita attiva: una mola, una pialla, dei chiodi, un martello, una squadra, una tenaglia e una sega. Il bel volto dell'angelo è immerso nell'ombra: solo riflettono la luce le sue lunghe vesti e una sfera immobile davanti ai suoi piedi. Alle sue spalle, si scorgono una clessidra, la cui sabbia sta correndo, una campana, una bilancia e un quadrato magico, e, sul mare che appare sullo sfondo, una cometa che brilla senza splendore. Su tutta la scena è diffusa

Io. Ibid., p. 76 sq. 
un'atmosfera crepuscolare, che sembra togliere a ogni particolare la sua materialità ${ }^{11}$.

L'angelo dell'arte interviene quando la tempesta del progresso si placa e anche gli utensili della vita attiva tacciono (come se fossero carichi di un potenziale di estraneazione). Il suo sguardo, immerso in una atmosfera intemporale, è immobile ma guarda davanti a sé (non al passato come l'angelo della storia). Secondo Agamben:

[...] il passato che l'angelo della storia ha perso la capacità di comprendere ricompone davanti all'angelo dell'arte la sua figura; ma questa figura è l'immagine estraniata in cui il passato ritrova la sua verità solo a condizione di negarla e in cui la conoscenza del nuovo è possibile solo nella non-verità del vecchio ${ }^{12}$.

La redenzione che l'angelo offre al passato, citandolo a comparire fuori del suo contesto, è la sua morte nel museo dell'esteticità.

Applicato al paesaggio questo significa che l'estetizzazione del paesaggio è la coscienza di un'estraneazione e la nostaglia di una realtà che non si possiede più. Qualcosa che non ci può bastare ma dalla cui consapevolezza non possiamo non ripartire anche per andare oltre. Anche per ricostruire il nostro rapporto con il paesaggio.

La nostalgia, come ho cercato di dire nel Rapporto sul paesaggio intitolato I paesaggi italiani fra nostalgia e trasformazione ${ }^{13}$, non è un sentimento sterile: al contrario, come riconosce anche Agamben, attraverso la bellezza si apre all'uomo uno spazio fra passato e futuro in cui egli può fondare la sua azione e la sua conoscenza. Ma questa via richiede che si restauri un corretto, positivo rapporto con la storia, basato magari sull'ironia - di cui oggi, a più di 50 anni dalla tragica morte di Benjamin, dobbiamo essere capaci senza cadere nelle spire del più vacuo postmodernismo. Un buon modello ci è offerto dall'angelo inventato da un artista poliedrico come Tonino Guerra. L'angelo coi baffi ${ }^{14}$ è la creatura ironica che «invece di volare attorno al Signore» si mescola

\footnotetext{
II. G. Agamben, L'uomo senza contenuto, p. 164.

I2. Ibid., p. 165.

I3. Rapporto annuale 2009: M. Quaini (a cura di), I paesaggi italiani tra nostalgia e trasformazione.

I4. T. Guerra, La valle del Kamasutra, p. 408 sq.
} 
alla vita degli uomini e al paesaggio e, per un rapporto più positivo con il passato e con il futuro, nutre il sogno impossibile di ridare vita agli oggetti estraniati e impagliati nel museo, nei nostri musei personali o collettivi che siano.

Anche se "tutti i santi ridevano" di lui e della sua pretesa di dare da mangiare agli uccelli impagliati, questi «una mattina hanno aperto le ali e hanno preso il volo, fuori dalla finestra e dentro l'aria del cielo e cantavano come non mai», come si conclude la poesia.

Tonino Guerra, grazie alla forza della poesia e della sua arte scenografica, ha fatto del Montefeltro un grande, straordinario museo all'aperto, che vive in un rapporto positivo con il passato perché vive nel presente e nel futuro, lo costruisce pezzo per pezzo. Perché la sua arte, i suoi progetti, nel realizzarsi a scala locale, non sono mai separati dalla quotidianità del lavoro e dalla vita delle società locali di cui sono strumento di alfabetizzazione storica e paesaggistica e di valorizzazione del patrimonio e delle risorse ambientali.

Le figure umane di cui parlano i disegni e le poesie di Guerra non sono immobili e meditabonde come Malinconia. Tanto meno lo è lui. Il «miracolo» di Guerra è nel suo sguardo di poeta e di eterno bambino che sa ancora meravigliarsi e "accontentare i desideri oscuri della memoria", sa ritrovare "la saggezza e l'immaginazione infantile e folle" che "come frutti di un orto abbandonato" nascono nei "continenti sepolti di gente anziana che vive in solitudine» nei borghi e nelle case del Montefeltro ${ }^{15}$.

Se un collegamento verrebbe di fare è ancora con Benjamin e con la sua visione del mondo messianico - «il mondo di una totale e integrale attualità " - nel quale soltanto diceva che si realizza per la prima volta una "storia universale» e che questa si doveva tradurre un una lingua di tutti «ma non come scritta, piuttosto come festosamente celebrata». Il linguaggio di questa festa «è l'idea della prosa stessa, che è compresa da tutti gli uomini, come la lingua degli uccelli dai nati di domenica» ${ }^{16}$.

L'interpretazione di questa antica leggenda ebraica non è facile, ma con l'aiuto di Tonino Guerra possiamo forse riconoscere in questa festa il paesaggio, proprio in quanto il suo linguaggio può essere compreso da tutti. Il paesaggio, dunque, come "prosa liberata» dalle catene

I5. T. Guerra, La valle del Kamasutra, p. 421 sq.

I6. G. Scholem, Walter Benjamin e il suo angelo. 
della scrittura e della carta (anche quella geografica), come festa di una liberazione anche sociale e politica. L'aveva già detto Rousseau ed è stato ripetuto dal filosofo Dagognet in un famoso convegno che si interrogava sulla morte del paesaggio ${ }^{17}$ :

Il paesaggio ci arriva attraverso e con la morte dei giardini. Il teatro della natura e dei campi, che appartiene a tutti, era stato perimetrato e interdetto nell'antico regime. J.J. Rosseau festeggia l'assenza di appropriazione, un patrimonio da condividere [...]. Allora si apre, con il paesaggio, la dialettica della festa di un bene sottratto a una lunga confisca, quella di un fuori che sfugge alla prigionia di un dentro troppo inclusivo, quella di un piacere a un tempo universale (anche comunitario) e inesauribile (la soddisfazione non distrugge più ciò che essa gusta e sente) ${ }^{18}$.

È per questa via che il territorio-paesaggio, sempre che riesca a mantenere questo senso, deve oggi tornare ad associarsi ad un'utopia che ho chiamato conviviale, in quanto fondata sulla scoperta del godimento di un bene comune sottratto a una lunga confisca o privatizzazione e, secondo Dagognet, su una "soddisfazione che non distrugge più ciò che essa gusta e sente» ovvero che attiva tutti i nostri sensi. Queste mi sembrano oggi le coordinate e le dimensioni politiche che fanno del paesaggio un tema imprescindibile nella costruzione di una nuova cittadinanza e di una nuova governance.

Per avviarmi alla conclusione riprendo il filo della «lingua degli uccelli» e del tema annunciato dello spaventapasseri. Quale significato possiamo dare allo spaventapasseri per liberarlo dall'ipoteca terroristica, alla quale lo vuole costringere Franco Farinelli? Scorrendo il bel libro in cui Dario Lanzardo ${ }^{19}$ ha pubblicato i risultati della ricerca fotografica svolta in Piemonte, Liguria e Valle d'Aosta dal '94 al '97 e dove "sfilano i pupazzi che una minoranza di contadini continua a costruire con fantasia e creatività, per difendere semine e raccolte dagli uccelli » (pl. IV.2), mi sono convinto non solo che, come dice l'autore, questa sia «una preziosa tradizione da conoscere e conservare perché è il simbolo della permanenza di un radicamento alla terra e rimanda a un

17. F. Dagognet (dir.), Mort du paysage?

I8. Ibid., p. 9 sq.

19. D. Lanzardo, Spaventapasseri. 
tempo in cui il rapporto uomo-natura era più equilibrato" e anche che "l'immagine "terribile" dello spaventapasseri è più il risultato dell'idea di spavento che ha il contadino che non il frutto della sua esperienza contro gli uccelli» ${ }^{20}$. Infatti gli uccelli dialogano con gli spaventapasseri, spesso si posano sulle loro spalle, famigliarmente. Più degli uomini gli spaventapasseri sono come i bambini nati di domenica: capiscono il linguaggio degli uccelli.

Questo ci dicono l'arte e la letteratura quando prendono lo spaventapasseri come tema e ne rovesciano il senso come fa Casorati quando fa dello spaventapasseri il filo conduttore, tenuto in mano dagli uccelli, di una favola che mira avvalorare un'altra idea del rapporto uomo-natura. Quando anche gli spaventapasseri, simulacri umani, si fanno la guerra e soccombono (F. Casorati, Il grande duello, 1972; La morte del generale, 1973) e solo alla fine sembrano raggiungere un riscatto; quando, come uccelli, si elevano verso la luna, si liberano dalla prigione della carta che li inchiodava al suolo e alle pratiche della guerra o della lotta economica (Spaventapasseri volanti, 1973) ${ }^{21}$.

O come le coeve sculture che Angelo Ruga ha costruito nel paese di Mongreno nel Monferrato con una forte impronta espressionista e un forte valore di denuncia dei valori o disvalori dominanti : in esse gli spaventapasseri, come nel romanzo straripante di Gunther Grass «non sono figure in conflitto con la natura umana o animale, ma ne simboleggiano la sconfitta a opera dell'uomo»"22. Anni di cane ${ }^{23}$ di Günter Grass è il romanzo in cui lo spaventapasseri e la sua fabbricazione diventa la metafora più ricca per capire la storia del Novecento e gli approdi della nostra malintesa modernità.

Infine non è un caso se anche Lanzardo, al quale devo lo stimolo iniziale a intraprendere questo percorso, approdi, a conclusione del suo lavoro interpretativo di fotografo curioso e intelligente, al tema dell'antropomorfismo paesaggistico dal quale anch'io sono partito e veda in esso, cioè nella capacità di riconoscere forme umane in natura, «una forma primordiale di autoriconoscimento (autorappresentazione)

20. Ibid., p. 16 sq.

2I. Di questi disegni di F. Casorati si vedano le riproduzioni, accompagnate da interessanti considerazioni, in D. Lanzardo, Spaventapasseri, p. 36 sq.

22. Ibid., p. 43.

23. G. Grass, Hundejahre, 1963, traduz. it. di Enrico Filippini: Anni di cane, Milano, Feltrinelli, 2009. 
dell'uomo e quindi come una delle prime forme di identificazione della potenza divina nella propria immagine ${ }^{24}$.

\author{
Massimo QUaIni
}

Università degli Studi di Genova

24. D. Lanzardo, Spaventapasseri, p. 46. 


\section{BIBLIOGRAFIA}

Agamben, Giorgio, L'uomo senza contenuto, Macerata, Quodlibet, 1994 (1970).

Benjamin, Walter, Angelus novus: saggi e frammenti, traduzione e introduzione di Renato Solmi, Torino, Einaudi, 1962.

Cacciari, Massimo, L’Angelo necessario, Milano, Adelphi, 2003 (1986, edizione riveduta e ampliata).

Dagognet, François (dir.), Mort du paysage? Philosophie et esthétique du paysage, Actes du colloque de Lyon, 3-4 décembre 1981, Seyssel, Éditions Champ Vallon, 1982.

Grass, Günter, Hundejahre/Anni di cane, traduz. Enrico Filippini, Milano, Feltrinelli, 2009 (1963).

Guerra, Tonino, La valle del Kamasutra. Segni, sogni e altro scelti dal poeta, Milano, Bompiani, 2011.

Lanzardo, Dario, Spaventapasseri. Lo straccione divino, 150 fotografie a colori, Milano, Mondadori, 1997.

Quaini, Massimo (a cura di), I paesaggi italiani tra nostalgia e trasformazione, Roma, Società Geografica Italiana, 2009.

Scholem, Gershom, Walter Benjamin e il suo angelo, Milano, Adelphi, 1978.

Settis, Salvatore, Paesaggio, costituzione, cemento. La battaglia per l'ambiente contro il degrado civile, Torino, Einaudi, 2010.

Referenze iconografiche

Pl. IV.2:

Liliana Guazzo Lanzardo. 
